Annales Geophysicae (2002) 20: 445-460 (c) European Geophysical Society 2002

\title{
Energetic particle observations at the subsolar magnetopause
}

\author{
A. A. Eccles and T. A. Fritz \\ Boston University Center for Space Physics, Boston MA, USA \\ Received: 3 April 2001 - Revised: 25 June 2001 - Accepted: 9 July 2001
}

\begin{abstract}
The pitch-angle distributions (PAD) of energetic particles are examined as the ISEE-1 satellite crosses the Earth's magnetopause near the subsolar point. The investigation focuses on the possible existence of a particular type of distribution that would be associated with a source of energetic particles in the high-latitude magnetosphere. PADs, demonstrating broad, persistent field-aligned fluxes filling a single hemisphere (upper/northern or lower/southern), were observed just sunward of the magnetopause current layer for an extended period of many minutes. These distributions are a direct prediction of a possible source of energetic particles located in the high altitude dayside cusp and we present five examples in detail of the three-dimensional particle distributions to demonstrate their existence. From these results, other possible causes of such PADs are examined.
\end{abstract}

Key words. Magnetospheric physics (energetic particles, precipitating; magnetopause, cusp and boundary layers; magnetospheric configuration and dynamics)

\section{Introduction}

The recent discovery by the Polar satellite that the high altitude dayside cusp may be the location of an acceleration mechanism capable of producing appreciable fluxes of $100 \mathrm{~s}$ to $1000 \mathrm{~s}$ of $\mathrm{keV}$ ions from the shocked solar wind entering the cusp has been reported by Chen and Fritz (1998); Chen et al. (1997). The proposed acceleration process appears to be associated with diamagnetic cavities that are formed in the region of the cusp as the shocked solar wind is rammed into the cusp geometry. The resulting turbulence in the magnetic field that is produced most likely by the diverted kinetic energy of the flowing solar wind plasma is believed to be the responsible driver for the acceleration of ions in the cusp to radiation belt energies (Chen and Fritz, 1998). Many such cavities appear to be active at any given time, but each has an apparent lifetime of tens of minutes. As these cavities collapse, the energetic particles produced within a given cavity escape, after which they will then drift under the control of gradients and curvature effects in the geomagnetic field, or

Correspondence to: T. A. Fritz (fritz@bu.edu) move along open geomagnetic field lines and form a layer of such particles on the magnetopause (Fritz and Chen, 1999). The total magnetic field, $\mathrm{B}_{T}$, measured in these diamagnetic cavities is very depressed, ranging from values of a near zero field magnitude to values of about one-half of the surrounding fields, $B_{N C}(\mathrm{NC}=$ Near Cusp). In such a geometry, the cavity will provide large mirror ratios, $2.0<B_{N C} / B_{T}<\infty$, which means that an effective trap is formed. Since the magnetosphere is compressed by the solar wind at the dayside equatorial plane, the minimum magnetic field along a field line is in the high-latitude region surrounding the cusp and not at the usual location of the equatorial plane. As energetic particles move along such field lines toward the equator, they will be observed to fill a full hemisphere with particle pitch-angles from field-aligned to locally mirroring pitchangles at $90^{\circ}$ but not very much beyond if the field lines are open. Such particles have been reported on the flanks of the magnetosphere appearing on both sides of the magnetopause (Williams et al., 1985; Mitchell et al., 1987). Many authors have reported observations of energetic particles in the magnetosheath near the magnetopause (Haskell, 1969; Meng and Anderson, 1970; Sarris et al., 1976; Baker and Stone, 1978). An obvious prediction of the concept of such a cusp source would be that energetic ions should be observed coming preferentially from either the northern or southern cusp direction, as a satellite penetrates the subsolar magnetopause, moving from the closed field line geometry of the trapping region to the open field line configuration in the magnetosheath associated with a rotational discontinuity at the magnetopause. The question to be addressed by this study will be: "are broad hemispheric distributions of energetic particles centered on a single field-aligned direction seen just outside the magnetopause?"

\section{Instrumentation and data selection}

The data set used is from the ISEE-1 spacecraft which was launched together with ISEE-2 on 22 October 1977 into an elliptic orbit with an apogee of $23 R_{E}$, a perigee of $280 \mathrm{~km}$, a period of about $57 \mathrm{~h}$, and an inclination of about $28^{\circ}$. A variable distance separated the two spacecraft, so that data from complementary instruments can be used to separate space- 

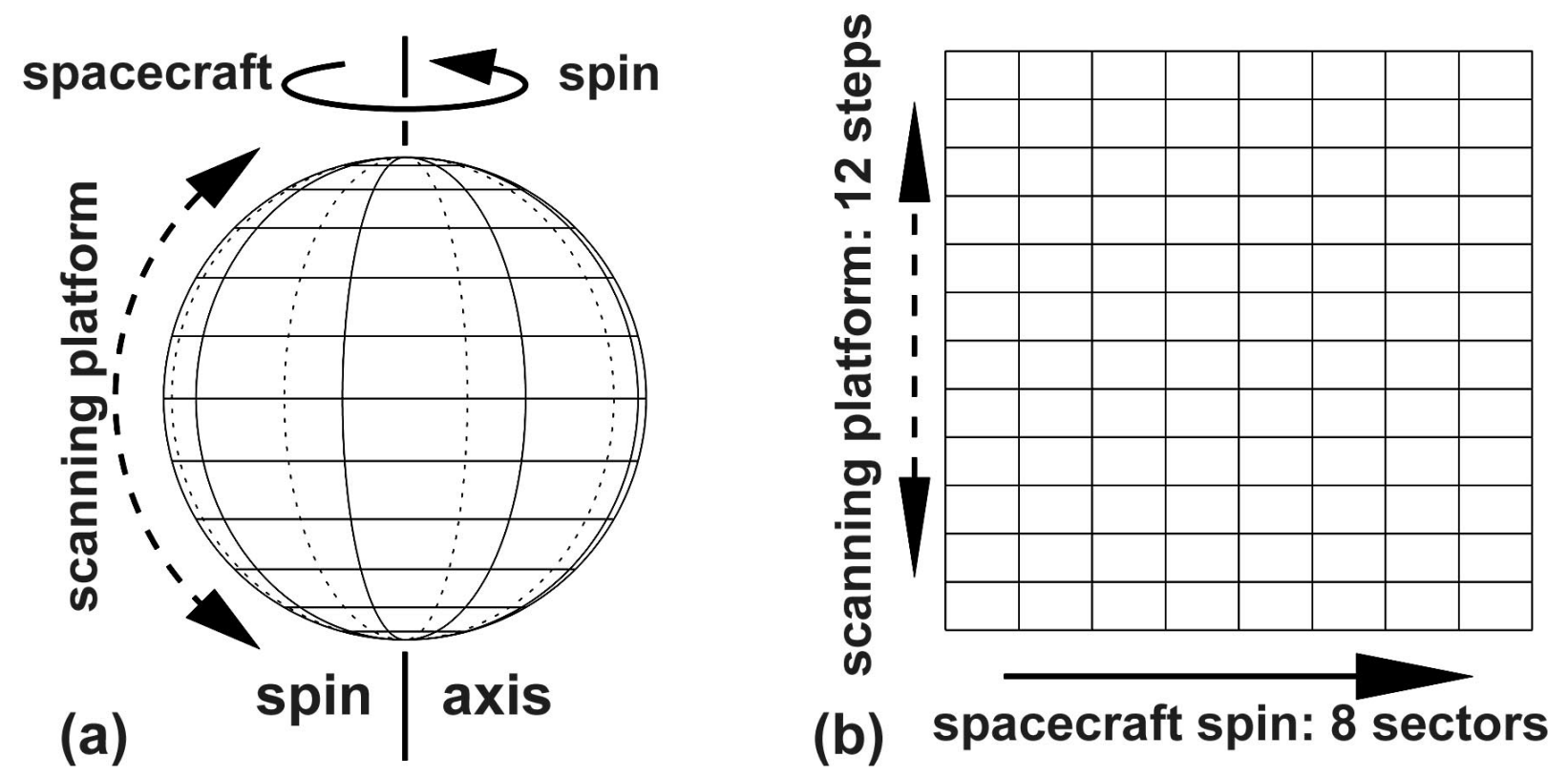

Fig. 1. (a) The measurement of all directions on the unit sphere is produced during one $36.5 \mathrm{~s} \mathrm{spin} / \mathrm{scan}$ cycle of the WAPS instrument on board ISEE-1. (b) The unit sphere can be made into a mercator projection, on which pitch-angle contours and flux intensities are overlaid. The resulting plot is a pitch angle distribution (PAD) angle/angle plot.

time ambiguities. The Medium Energy Particles Experiment (MEPE) on ISEE-1 and 2 was designed to detect electrons and ions ranging from $22.5 \mathrm{keV}$ to $1.2 \mathrm{MeV}$ for electrons, and from $24 \mathrm{keV}$ to $2 \mathrm{MeV}$ for protons. These energy ranges were divided into eight energy channels, each for electrons and ions. The WAPS (Wide Angle Particle Spectrometer) on ISEE-1 was mounted on a scan platform which rotated $160^{\circ}$, from almost parallel to almost antiparallel to the spin axis in 12 satellite spin periods which required about $36.5 \mathrm{~s}$. Williams et al. (1978) have given detailed descriptions of this instrument. The data returned from the ISEE-1 MEPE were converted to flux for each of the energy passbands and plotted as a function of both time and L-value (McIlwain, 1961) for the two years of operational life of the ISEE-1 experiment. Using simultaneous measurements of the geomagnetic field provided by the onboard magnetometer (Russell et al., 1978), the instantaneous pitch-angle of a given particle measurement was determined. As illustrated in Fig. 1 for each $36.5 \mathrm{~s} \mathrm{spin} / \mathrm{scan}$ cycle of the WAPS, a representative flux corresponding to each energy channel for eight sectors of each spin was determined. This resulted in 96 samples being made on the $4 \pi$ steradian unit sphere. These fluxes could be displayed in a colour-coded manner on a mercator projection of the unit sphere, where the vertical axis is the polar angle and the horizontal axis is the azimuthal angle in spacecraft coordinates which were essentially Geocentric Solar Ecliptic (GSE) coordinates. The scale on the horizontal axis $(\phi)$ has the sunward direction at $0^{\circ}$ and the $180^{\circ}$ direction is antisunward. For clarity, it is emphasized that the look direction is the direction from which the energetic particles are coming. The vertical axis direction $(\theta)$ is the scan direction where $\theta=0^{\circ}$ is antiparallel to the spacecraft spin axis viewing the south ecliptic pole, and $\theta=180^{\circ}$ is parallel to the spin axis viewing the north ecliptic pole. At $\theta=90^{\circ}$, the $0^{\circ}$ and $90^{\circ}$ azimuthal directions represent essentially the GSE $X$ - and $Y$-axes, respectively. The pitch-angle associated with each flux measurement was determined instantaneously from the magnetometer data and used to place the contours of constant pitch-angle on these unit sphere angle/angle $(\theta, \phi)$ displays. From a list of ISEE-1 magnetopause crossings supplied by S. Petrinic (private communication), MEPE WAPS data were selected for a three-hour period centered on magnetopause crossings over a range of dayside local times. The particle flux intensity variations as a function of sensor look direction in each angle-angle plot produced a three-dimensional particle distribution for each $36.5 \mathrm{~s}$ spin-scan cycle. After examining each plot, a particle distribution code was assigned to it. It was found that all ion distributions could be placed into one of eleven categories. Figure 2 is a listing of these categories, each of which is accompanied by its respective description, number, assigned colour-code, and an example taken from the ISEE-1 data. This study will ask the straightforward question as to whether the distributions in Fig. 2, indicated by the numbers five and six, occur and if so, how frequently in a given pass and what is their relationship to the position of the magnetopause.

As a point of information about the ISEE-1 MEPE, the instrument responses from the WAPS sensor were accumulated in a manner that was synchronized to the satellite spin period and not the satellite telemetry cycle. Occasionally, the amount of information accumulated for read-out into the telemetry would exceed the bit rate of the telemetry allocation and a full spin/scan cycle would be lost. The MEPE was also sensitive to an on board radio frequency plasma sounder 


\begin{tabular}{|c|c|c|c|c|}
\hline Distribution Type & Description & No. & Color & Example \\
\hline Background & $\begin{array}{l}\text { Flux intensity throughout the panel was } \\
\text { extremely low or zero and displayed a } \\
\text { random pattern. }\end{array}$ & 0 & & \\
\hline Isotropic & Smooth flux intensity throughout. & 1 & & \\
\hline Trapped & $\begin{array}{c}\text { Peak in flux intensity at a } 90 \text {-degree pitch } \\
\text { angle. }\end{array}$ & 2 & & \\
\hline Non-Gyrotropic & $\begin{array}{l}\text { Azimuthal asymmetries at 90-degree } \\
\text { pitch angle. }\end{array}$ & 3 & & \\
\hline Butterfly & $\begin{array}{l}\text { A minimum in flux intensity at 90-degree } \\
\text { pitch angle. }\end{array}$ & 4 & & \\
\hline $\begin{array}{c}\text { Northern } \\
\text { Hemispheric Flow }\end{array}$ & $\begin{array}{l}\text { Flow of particles from the north, filling } \\
\text { the hemisphere. }\end{array}$ & 5 & & \\
\hline $\begin{array}{c}\text { Southern } \\
\text { Hemispheric Flow }\end{array}$ & $\begin{array}{l}\text { Flow of particles from the south, filling } \\
\text { the hemisphere. }\end{array}$ & 6 & & \\
\hline $\begin{array}{l}\text { Dusk-Side } \\
\text { Beaming }\end{array}$ & $\begin{array}{l}\text { Field aligned flow from the subsolar } \\
\text { point toward the dusk side of the } \\
\text { magnetosphere. }\end{array}$ & 7 & & \\
\hline $\begin{array}{l}\text { Dawn-Side } \\
\text { Beaming }\end{array}$ & $\begin{array}{l}\text { Field aligned flow from the subsolar } \\
\text { point toward the dawn side of the } \\
\text { magnetosphere }\end{array}$ & 8 & & \\
\hline $\begin{array}{c}\text { Weak or } \\
\text { Disturbed } \\
\text { Magnetic Field }\end{array}$ & $\begin{array}{l}\text { Magnetic field was disturbed or } \\
\text { weakened as evident by the irregularity } \\
\text { with no obvious particle distribution. }\end{array}$ & 9 & & \\
\hline $\begin{array}{l}\text { Apparent } \\
\text { Beaming }\end{array}$ & $\begin{array}{l}\text { Flow of particles seeming to ignore field } \\
\text { direction. }\end{array}$ & 10 & & \\
\hline Bad Data & Bad or missing data. & 11 & & \\
\hline
\end{tabular}

Fig. 2. Examples and short descriptions of the eleven types or categories of PADs with an indication of the numerical and colour-code associated with the respective PADs. 

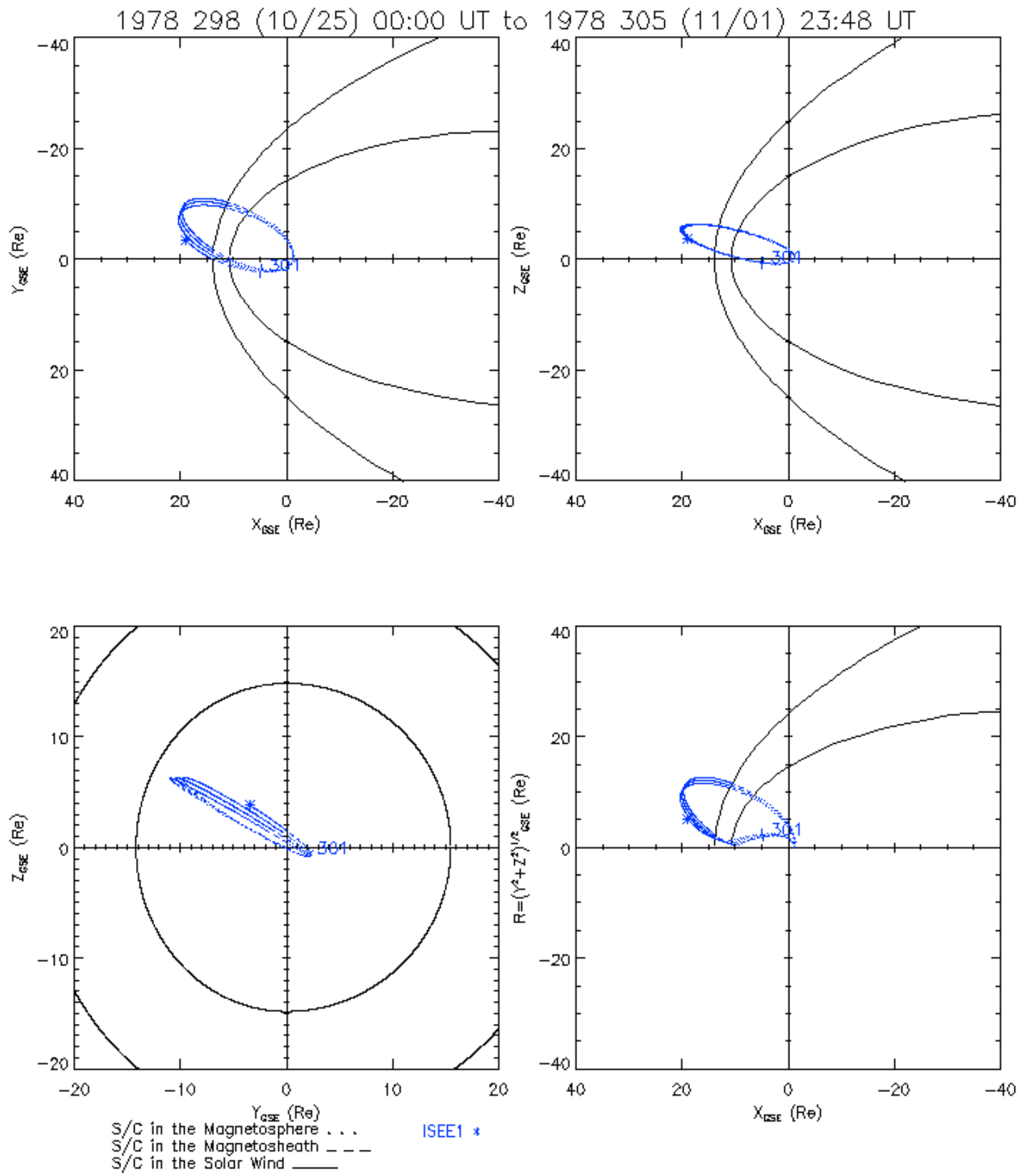

Generated by SSCweb on: Wed Jun 13 14:39:34 2001

Solar Wind Pressure $=2.1 \mathrm{nP}$ IMF BZ=0.0nT

Fig. 3. Orbit of ISEE-1 during the period 25 October 1978 (Day 298) to 1 November 1978 (Day 305).

(Chris Harvey, PI) when it was operated in its active mode. At these times, noise would dominate the response of the lower energy channels and would mask these detector channel responses, particularly when the WAPS sensor was scanning directions that were nearly perpendicular to the satellite spin axis. The occurrences of either of these conditions were placed in category eleven of Fig. 2 and a blank panel was plotted in the $36.5 \mathrm{~s}$ angle/angle sequences and indicated by the black colour in the summary plots.

Five ISEE-1 passes through the magnetopause are presented here in detail. Three of these occurred near the subsolar region (12:00 LT) and the other two presented occurred near 8:00 LT. The geometry of the ISEE-1 orbit is presented in Fig. 3 for the first four cases considered below. 


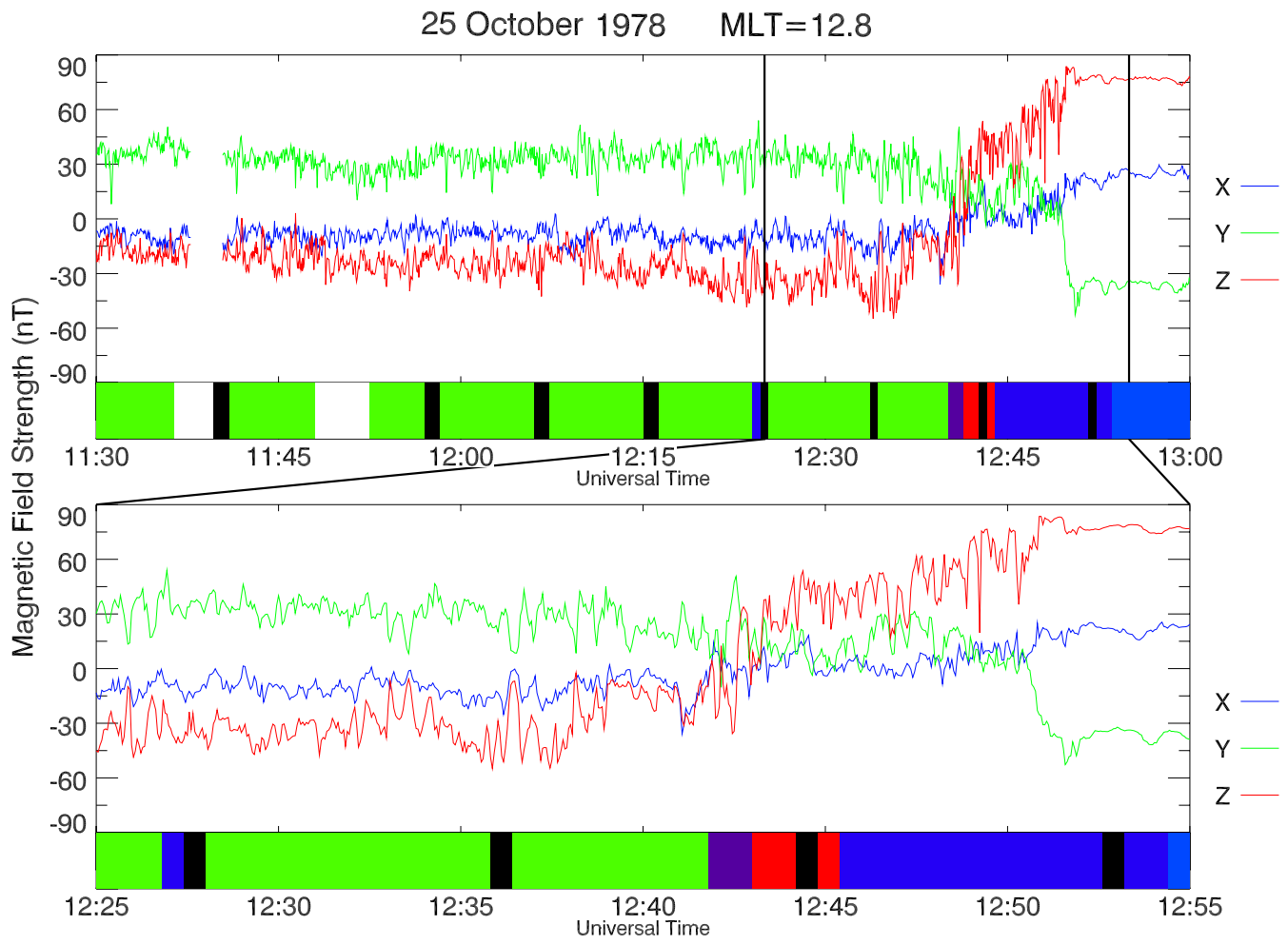

Fig. 4. The top plot shows the three components of the ISEE-1 measured magnetic field on 25 October 1978 (Day 298 ) from 11:30 to 13:00 UT. On the bar just below, the occurrence of various pitch-angle distributions (PADs) of the types presented in Fig. 2 is depicted. (Northern hemispheric PADs are those regions of green shading and the black shaded regions are periods of bad or missing data.) The bottom plot is a detailed blowup of the above plot from 12:25 to 12:55 UT.

Table 1. ISEE-1 positions at the beginning and end of the expanded time intervals of the magnetopause crossings. Note $X_{\mathrm{GSE}}=X_{\mathrm{GSM}}$

\begin{tabular}{|c|c|c|c|c|c|c|c|c|c|c|c|c|c|}
\hline \multirow[t]{2}{*}{ Fig. } & \multirow[t]{2}{*}{ Date } & \multirow{2}{*}{$\begin{array}{r}\text { Begin } \\
\text { UT }\end{array}$} & \multicolumn{3}{|c|}{$\operatorname{GSE}\left(R_{E}\right)$} & \multicolumn{2}{|c|}{$\operatorname{GSM}\left(R_{E}\right)$} & \multirow{2}{*}{$\begin{array}{c}\text { End } \\
\text { UT }\end{array}$} & \multirow[b]{2}{*}{$X$} & \multicolumn{2}{|c|}{$\operatorname{GSE}\left(R_{E}\right)$} & \multicolumn{2}{|c|}{$\operatorname{GSM}\left(R_{E}\right)$} \\
\hline & & & $X$ & $Y$ & Z & $Y$ & Z & & & $Y$ & Z & $Y$ & Z \\
\hline 4 & 25 Oct 1978 & $12: 25$ & 8.91 & 1.68 & 0.20 & 1.55 & -0.67 & $12: 55$ & 8.13 & 1.86 & 0.03 & 1.63 & -0.89 \\
\hline 6 & 28 Oct 1978 & 8:00 & 7.09 & -8.36 & 4.60 & -4.96 & 8.15 & 9:00 & 8.21 & -8.82 & 4.91 & -5.00 & 8.77 \\
\hline 8 & 30 Oct 1978 & $6: 25$ & 10.19 & 0.50 & 0.42 & 0.63 & 0.16 & $7: 05$ & 9.27 & 0.84 & 0.19 & 0.83 & -0.22 \\
\hline 10 & 1 Nov 1978 & $14: 25$ & 11.91 & -0.69 & 0.86 & -0.27 & 1.06 & $15: 25$ & 10.72 & -0.10 & 0.52 & 0.09 & 0.52 \\
\hline 12 & 18 Dec 1977 & $16: 00$ & 6.45 & -10.72 & 5.09 & -10.42 & 5.67 & $17: 00$ & 6.35 & -9.62 & 4.61 & -9.63 & 4.60 \\
\hline
\end{tabular}

\section{Case 1: Northern hemisphere on 25 October 1978 (Day 298)}

The first magnetopause crossing examined occured on an inbound pass on 25 October 1978 (Day 298). ISEE-1 was at a location of $12.8 \mathrm{LT}$ with GSE coordinates of $X=8.5 R_{E}, Y=1.8 R_{E}$, and $Z=0.1 R_{E}$ as the satellite crossed through the magnetopause (Fig. 3). When the detailed three-dimensional distributions are constructed for the period around the actual crossing, large fluxes of energetic protons completely filling the northern hemisphere of the local PAD were observed, while the southern hemisphere was entirely empty. ISEE-1 encountered the northern hemisphere type of PAD distribution continuously for more than an hour prior to the satellite encountering the magnetopause. Components of the magnetic field and a colour-coded index associated with the types of distributions that were presented in Fig. 2 are displayed in Fig. 4 for the interval from 11:30 UT to 13:00 UT. These distributions are determined from the particle fluxes measured by the P1 channel, which responded to ions of energy from 24 to $44 \mathrm{keV}$. An expanded version of the actual magnetopause crossing is given in the lower part of Fig. 4. The magnetosheath magnetic field had a negative $B_{Z}$ component, a positive $B_{Y}$ of similar magnitude and a small negative $B_{X}$ component. The IMF measured upstream at ISEE-3 had GSM components with the same directional sense, but the relative magnitude of $B_{X}$ was larger than $B_{Y}$. The magnetopause itself also required an extended time to 
P1 24.0 - $44.5 \mathrm{keV}$ lons 25 October 1978

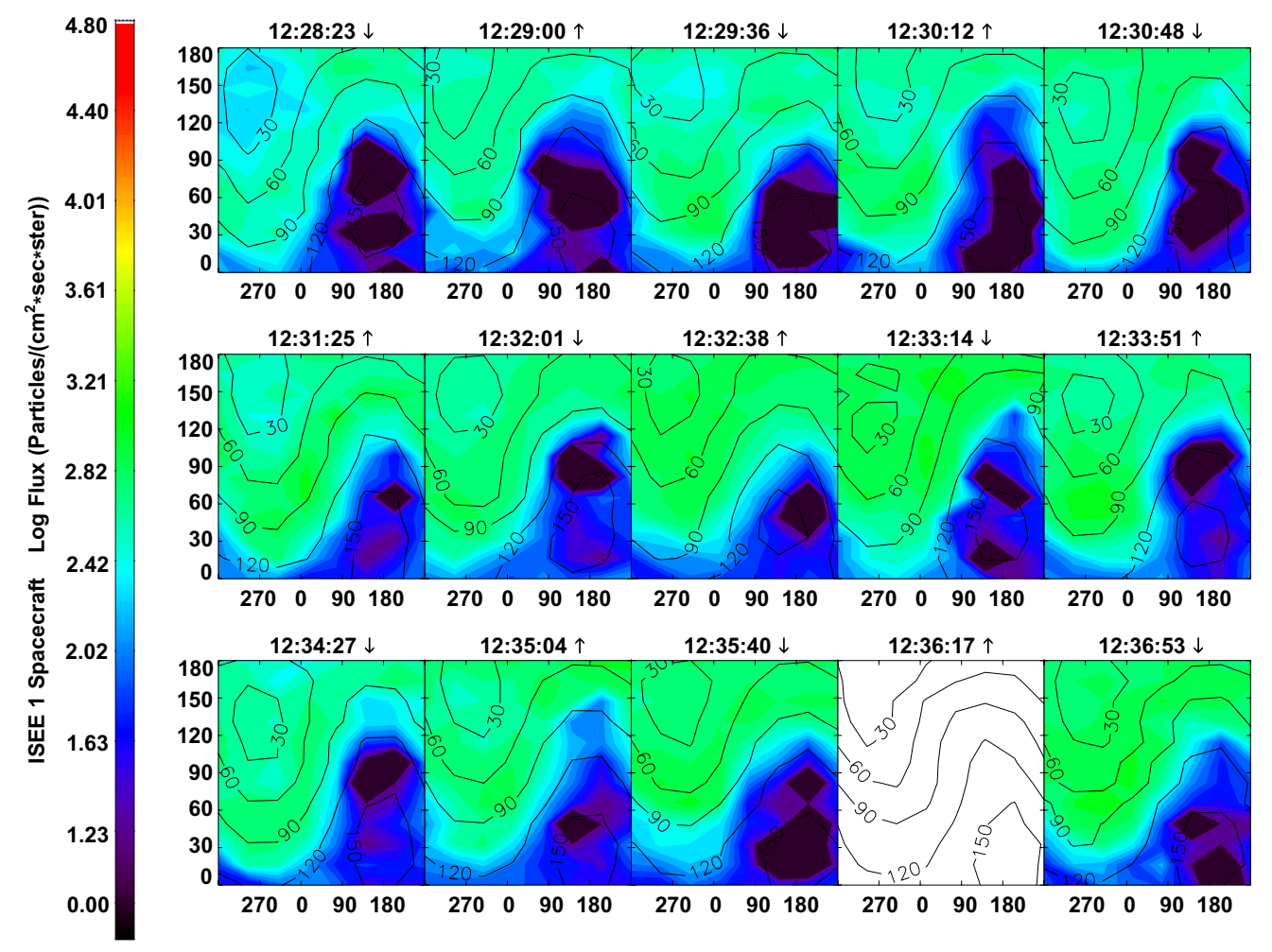

P1 24.0 - 44.5 keV lons 25 October 1978

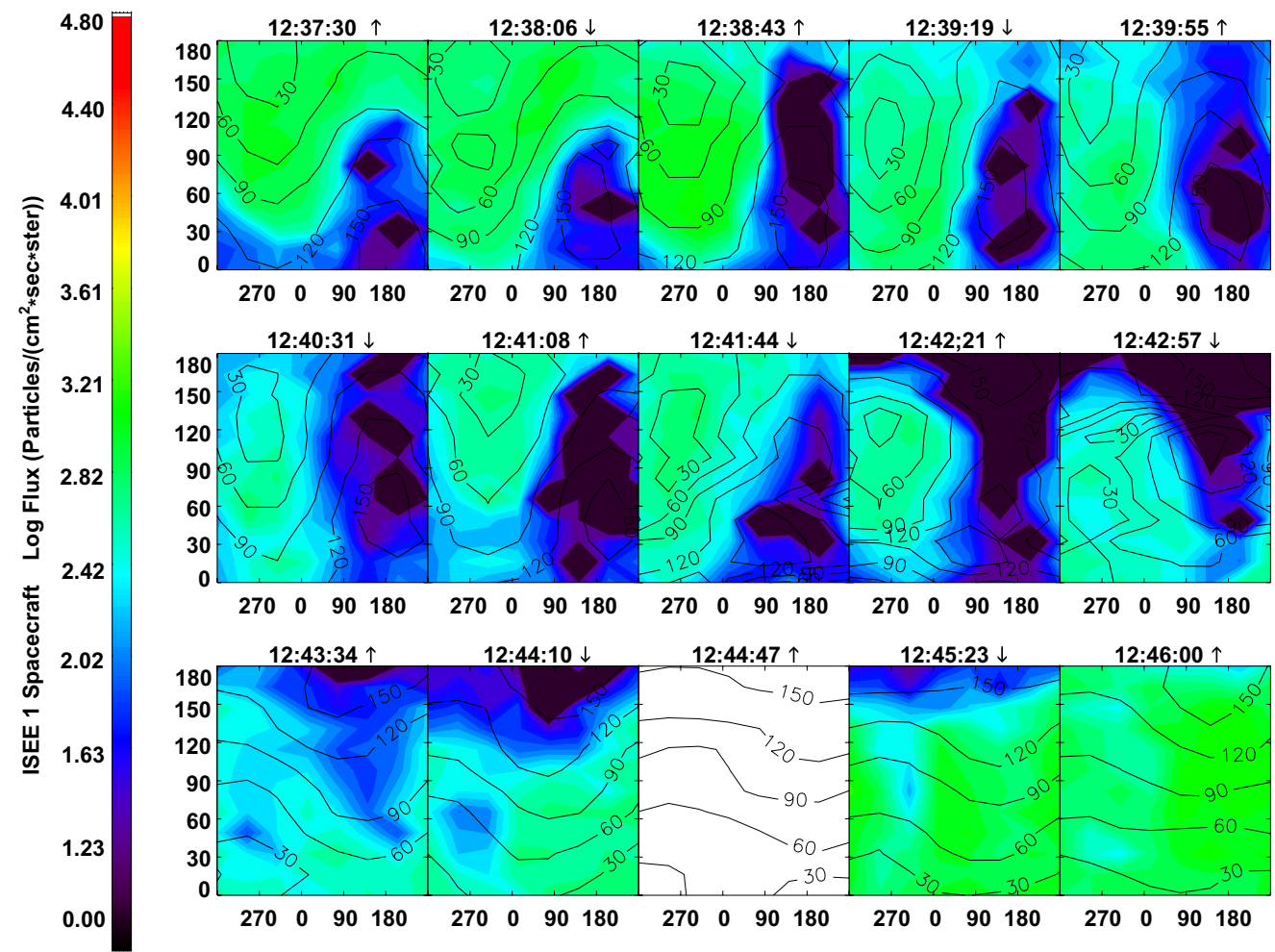

Fig. 5. A time sequence of three-dimensional PADs for the ISEE-1 Medium Energy Particles Experiment P1 channel which measured 24 to $44 \mathrm{keV}$ ions on 25 October 1978 (Day 298). Panel (a) shows a sequence of fifteen angle/angle plots from 12:28:23 UT to 12:36:53 UT demonstrating the northern hemispheric PADs. Panel (b) shows the next sequence of these plots from 12:37:30 UT to 12:46:00 UT in which the magnetopause current layer was crossed. The small arrows at the top of these angle/angle plots (and subsequent plots) indicate the direction of the vertical scan motion of the WAPS sensor. 


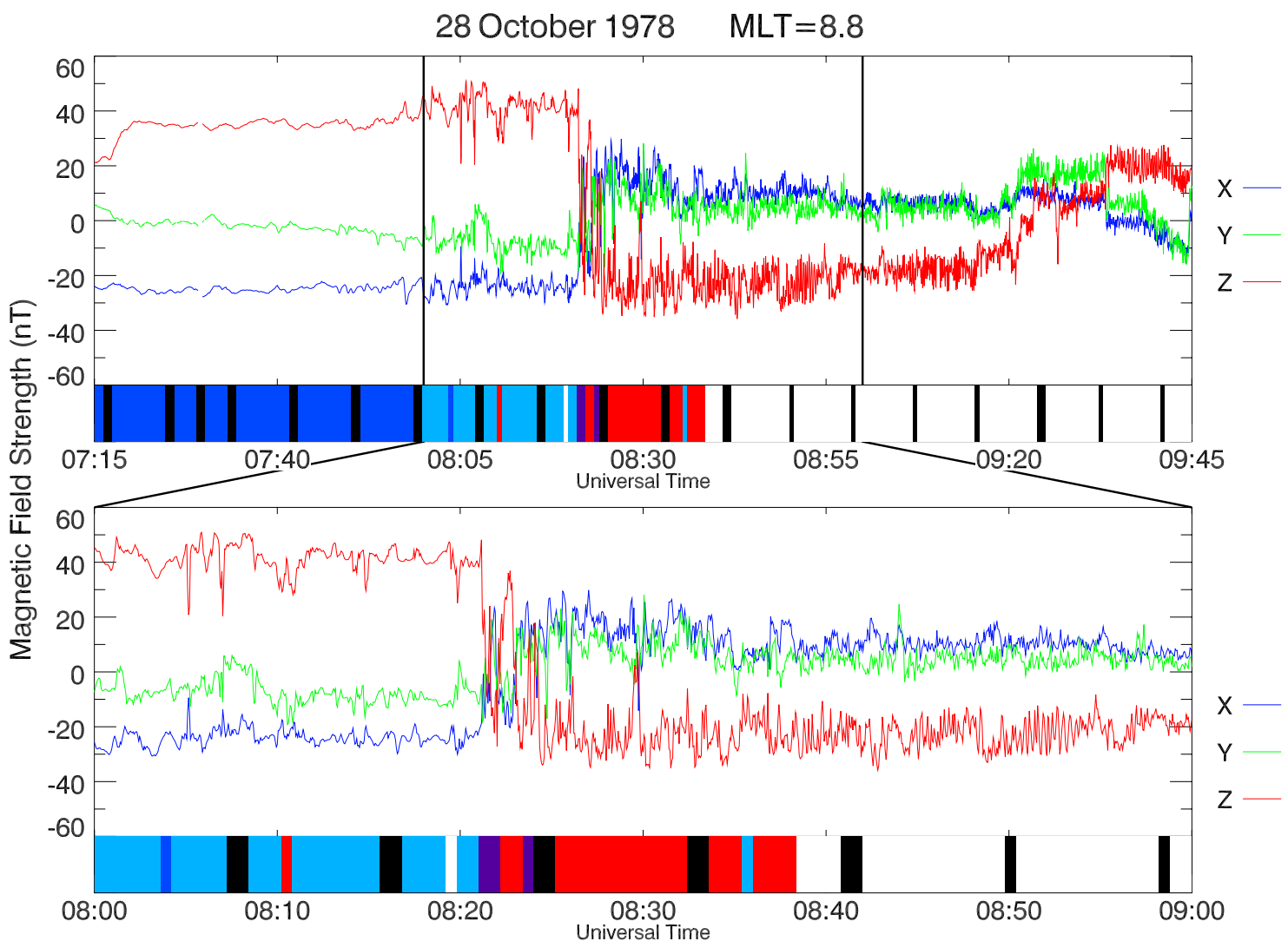

Fig. 6. A plot in the format of Fig. 4 for 28 October 1978 (Day 301) from 07:15 UT to 09:45 UT.

be crossed, more than $15 \mathrm{~min}$ from the initial rotation of $B_{Z}$ at 12:35 UT, until the steady magnetospheric field was encountered after 12:50 UT. A sequence of fifteen ISEE-1 angle/angle plots, each accumulated for $36.5 \mathrm{~s}$ during this interval, is shown in detail in Fig. 5, demonstrating the northern hemispheric PAD. (The panel of missing data at 12:36:17 UT was due to interference by the plasma sounder experiment noted in Sect. 2 above). The measured energetic particle PADs actually covered more than the upper hemisphere of the unit sphere, but still displayed a strong asymmetry, favoring particles arriving from the northern hemisphere. PADs of this type were observed contiuously for more than an hour. The energetic particles probably encountered a larger value of the absolute magnitude of the magnetic field further along the field line and a fraction of them would mirror away from the satellite and return along pitch-angle trajectories that would be measured at ISEE-1 as greater than $90^{\circ}$. Note that these northern hemispheric distributions were seen on and outside of the magnetopause. In Fig. 5b, note that the sense of the anisotropy changes at the magnetopause current layer, but that fluxes as a function of pitch-angle are maintained in the region just inside the current layer. This can be seen by noting that the $30^{\circ}$ and $60^{\circ}$ pitch-angle contours are filled, whereas the $120^{\circ}$ and $150^{\circ}$ contours are empty. This condition is maintained as the magnetic field and anisotropy reverses as the magnetopause current layer is crossed.

\section{Case 2: Southern hemisphere on 28 October 1978 (Day 301)}

The next case examined here, 28 October 1978 (Day 301), is an example of southern hemispheric PADs, and it occurred on the outbound portion of the ISEE-1 orbit (Fig. 3). During the period examined from 07:15 UT to 09:45 UT, as seen in Fig. 6, ISEE-1 appeared to have crossed out of the magnetosphere at approximately 8:21 UT. The satellite was located at $X=7.5 R_{E}, Y=-8.5 R_{E}$, and $Z=4.7 R_{E}$ with a local time of $8.8 \mathrm{~h}$. For the first $45 \mathrm{~min}$ of this period, the ISEE-1 MEPE WAPS sensor observed a completely trapped particle population. At approximately 08:00 UT, the magnetic field became disturbed as the spacecraft approached the outer edge of the magnetosphere. ISEE-1, however, remained inside the magnetopause until 08:21 UT since $B_{Z}$ was continuously positive. From 08:21 until 08:25 UT, the spacecraft passed through the current layer of the magnetopause. In the magnetosheath away from the magnetopause, ISEE-1 observed an IMF with a large negative value of $B_{Z}$ and small positive components of both $B_{X}$ and $B_{Y}$, as seen in Fig. 6 . There is a data gap in the measurements at ISEE-3 at this time, but they seem to reflect a steady solar wind velocity of $450 \mathrm{~km} / \mathrm{s}$ with the IMF having near zero values of $B_{X}$ and $B_{Y}$, and a negative value of $B_{Z}$, indicating a consistent steady structure to the large-scale IMF. Once in the magnetosheath, the ISEE-1 


\section{P1 24.0 - 44.5 keV lons 28 October 1978}
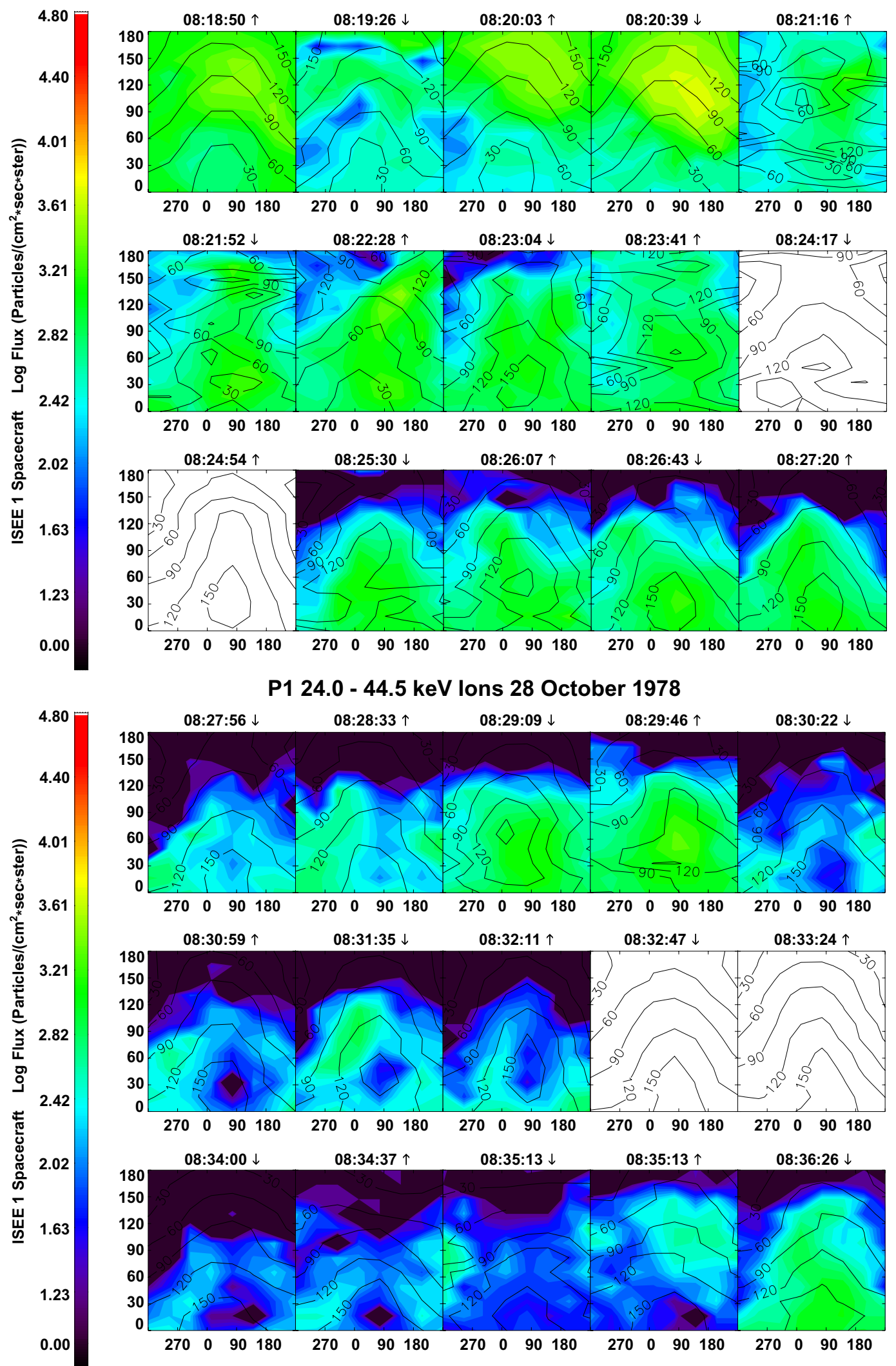

Fig. 7. A time sequence of southern hemispheric PADs for 24 to $44 \mathrm{keV}$ ions on 28 October 1978 (Day 301) beginning from (a) 08:18:50 UT to (b) ending at 08:36:26 UT for the ISEE-1 Medium Energy Particles Experiment P1 channel. 


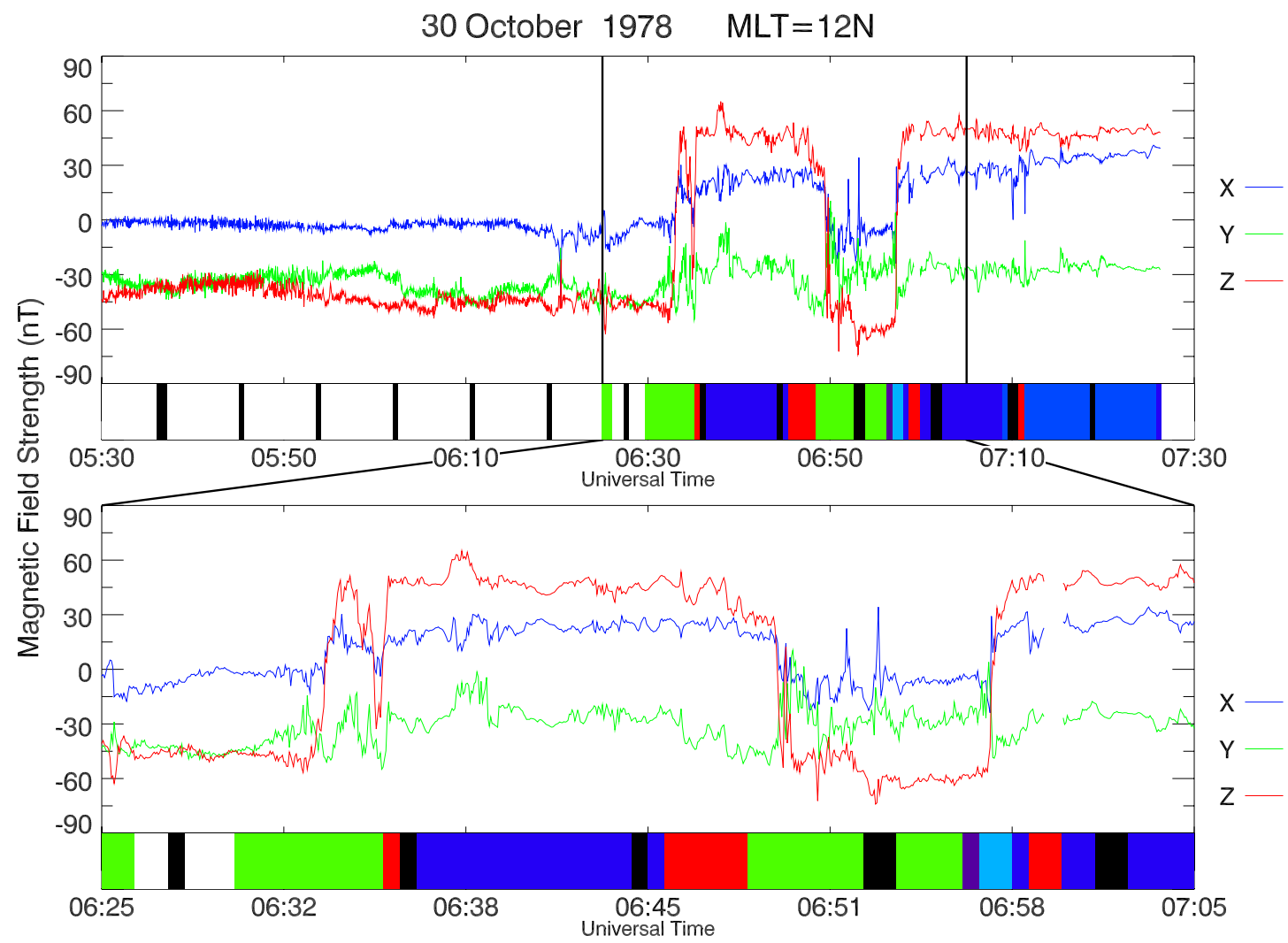

Fig. 8. A plot in the format of Fig. 4 for 30 October 1978 (Day 303) from 05:30 UT to 07:30 UT.

satellite observed a nearly continuous flux of energetic particles streaming from the south, which lasted from 08:25 to 08:39 UT, nearly $15 \mathrm{~min}$. A fifteen panel sequence of this interval is presented in Fig. 7. After the southern hemispheric flux of ions diminished, only background fluxes were observed. Note that these southern hemispheric distributions were seen just outside the magnetopause.

\section{Case 3: Northern hemisphere on 30 October 1978 (Day 303)}

On the following inbound pass from that presented as Case 2, there were again intervals of northern hemispheric PADs observed. The location of the satellite with respect to the magnetopause is presented in Fig. 8 for the period between 05:30 and 07:30 UT on 30 October 1978 (Day 303). Upstream at ISEE-3, the IMF and solar wind were steady. The GSM $B_{X}$ component of the IMF was small and the other two components were negative in a total field of $10 \mathrm{nT}$. The solar wind velocity was around $400 \mathrm{~km} / \mathrm{s}$. ISEE- 1 was inbound and encountered the magnetopause on at least 5 occasions, with the first occurrence at about 06:33 UT. The crossing was at local noon with GSE coordinates of $X=10.0 R_{E}$, $Y=0.6 R_{E}$, and $Z=0.4 R_{E}$ (Fig. 3). At that time, the magnetic field measured at ISEE-1 abruptly turned northward. Before that point, the field was nominally south- ward with $B_{Z} \sim B_{Y} \sim-45 \mathrm{nT}$ from 06:30 to 06:32 UT and from 06:34 to 06:35 UT, the $B_{Z}$ component was approximately $+45-50 \mathrm{nT}$. A sequence of 15 ISEE-1 angle/angle plots that occurred from 06:26:09 UT to 06:34:39 UT is presented in Fig. 9a, in which the period of the first magnetopause crossing is included. While ISEE-1 was upstream of the magnetopause in the magnetosheath, the PAD filled the unit sphere out to pitch-angles of $90^{\circ}$ over the upper hemisphere continuously for more than $5 \mathrm{~min}$. As the magnetopause was approached and the field underwent the rotation described above, the energetic particles surprisingly appeared to ignore the field change initially and maintained the northern hemisphere PAD before giving way to distributions found inside the magnetosphere. In Figs. $9 \mathrm{~b}$ and $9 \mathrm{c}$, the individual PAD panels are presented for the period from 06:43:46 UT to 07:01:23 UT. This sequence covers two magnetopause crossings with ISEE-1 first moving into the magnetosheath at 06:49 UT and crossing back into the magnetosphere at 06:57 UT. While in the magnetosheath, ISEE-1 observed the full or partial broad northern hemispheric PAD. In these two crossings, the anisotropy reversed, with the reversal of the magnetic field across the magnetopause current layer, indicating that the direction of the energetic particles followed the field direction even during reversals. Again, note that these northern hemispheric PADs were observed on or just outside the magnetopause in each case. 


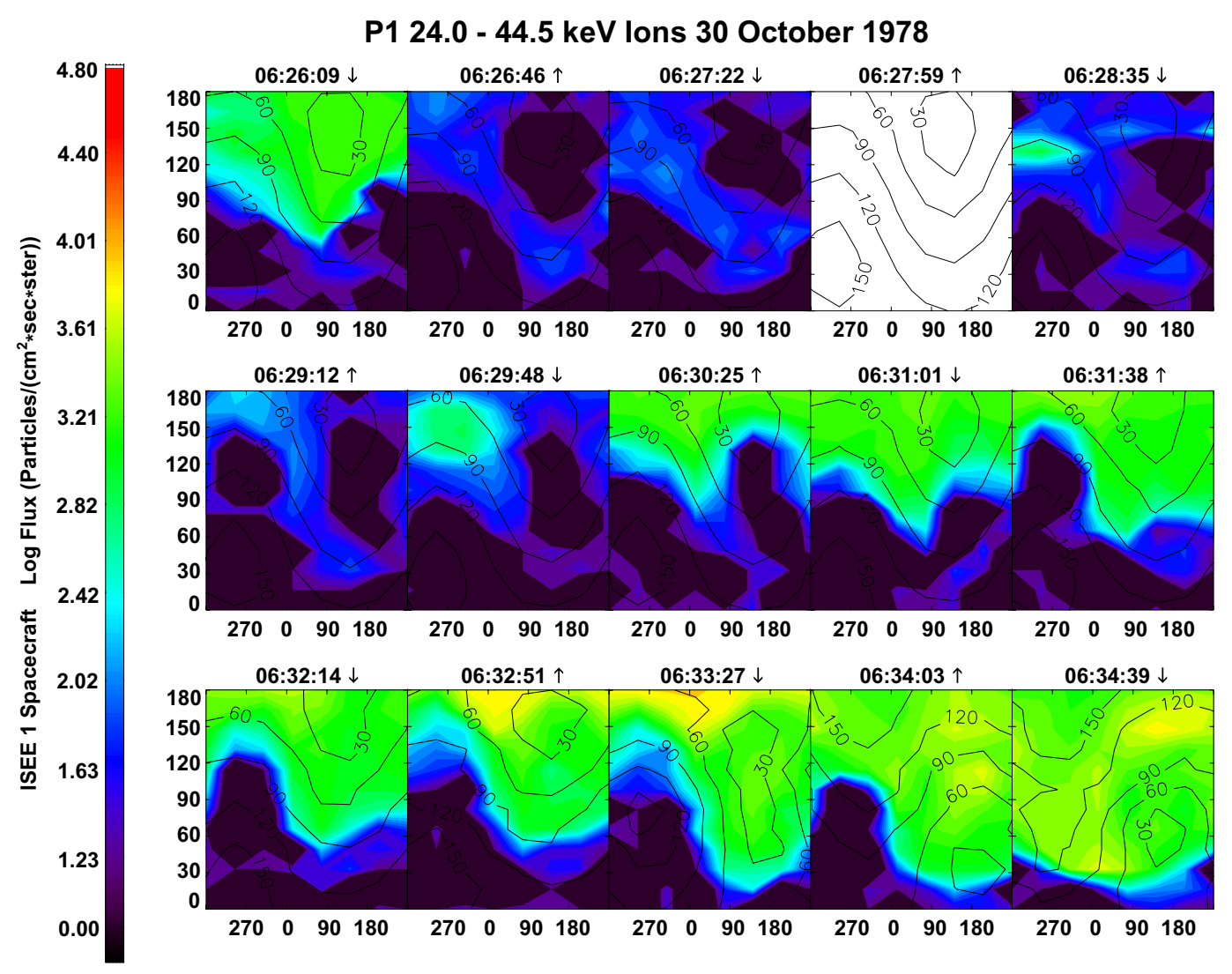

\section{P1 24.0 - 44.5 keV lons 30 October 1978}
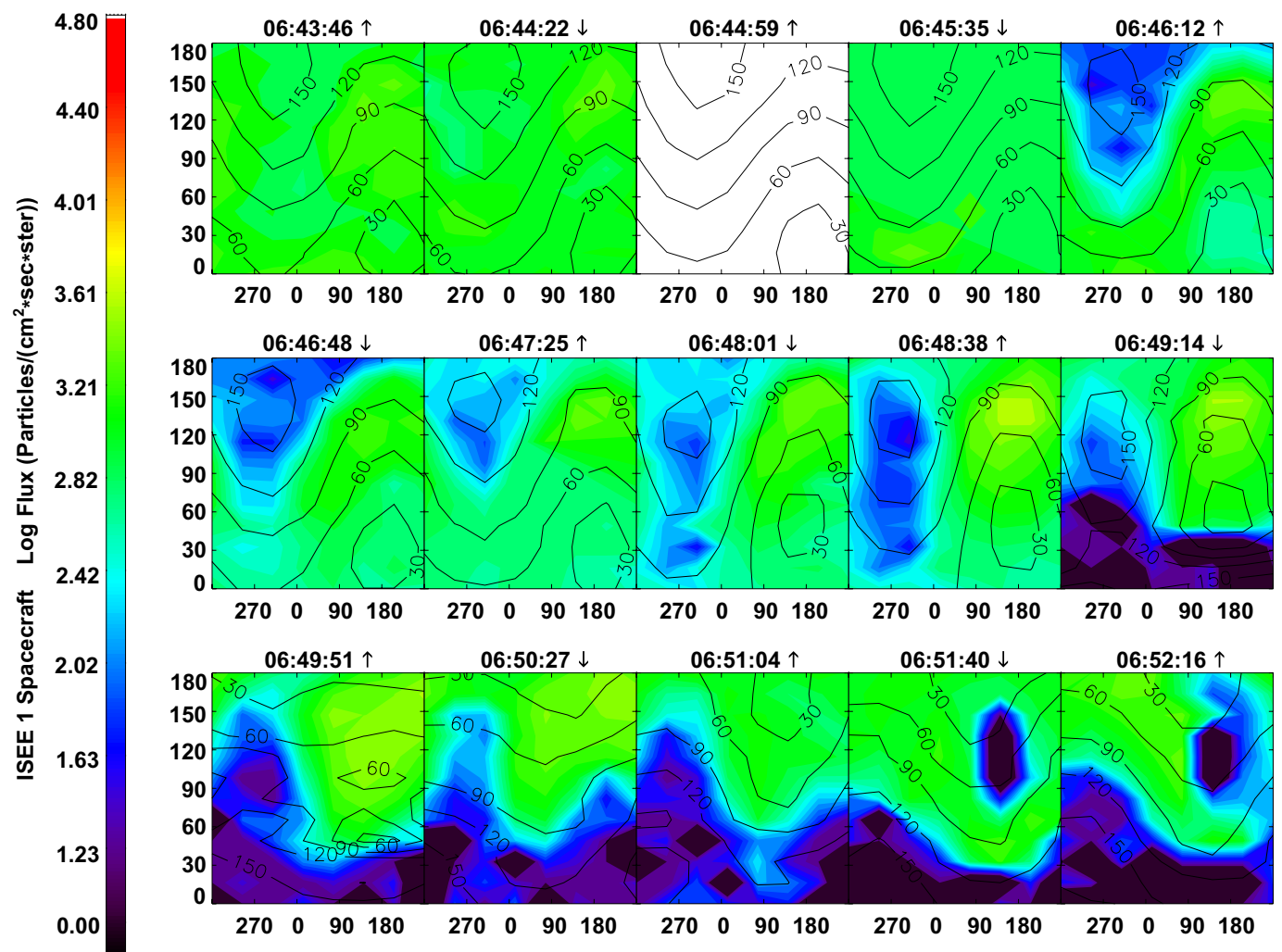

Fig. 9. continued 


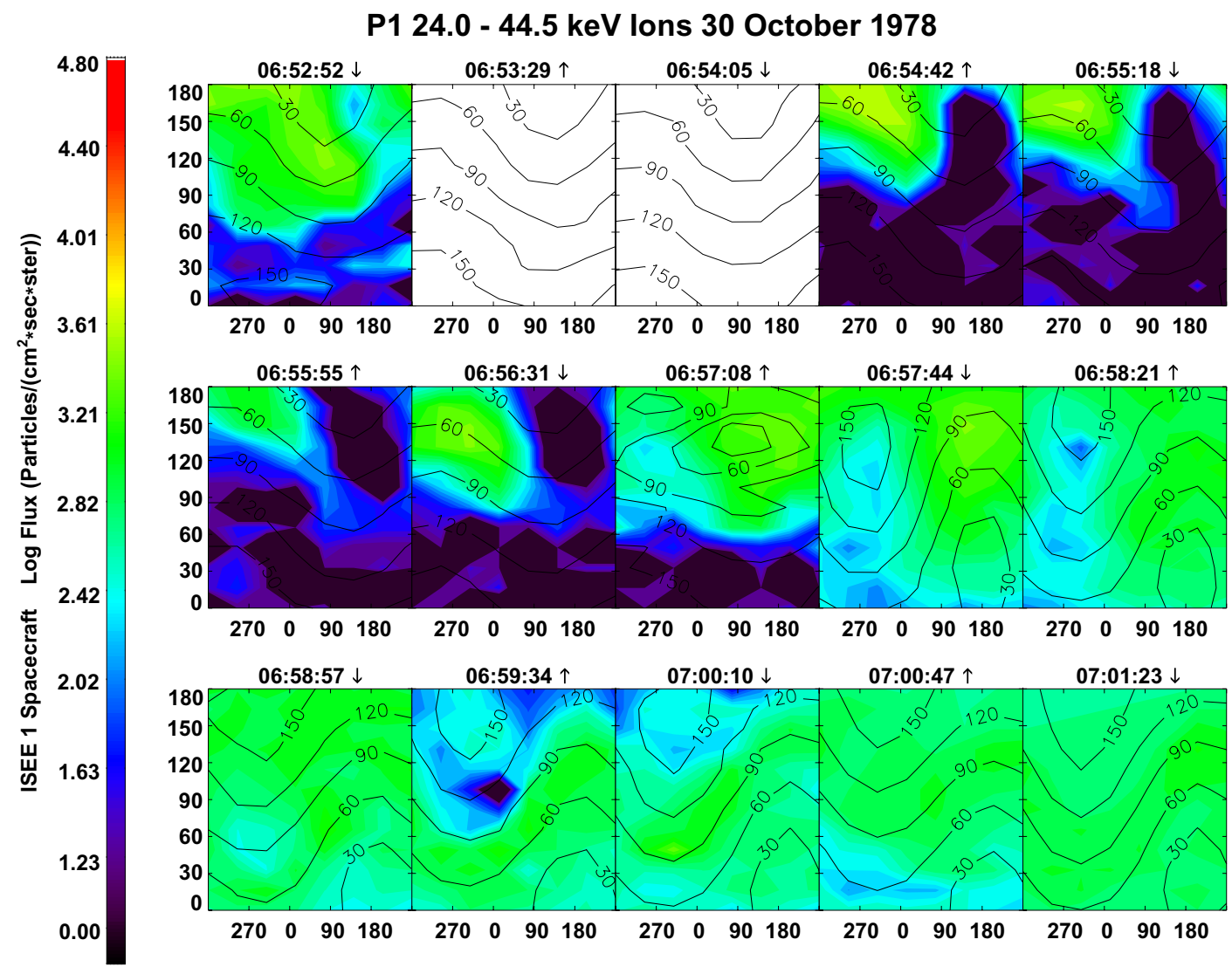

Fig. 9. A time sequence of northern hemispheric PADs for 24 to $44 \mathrm{keV}$ ions on 30 October 1978 (Day 303) from (a) 06:26:09 UT to 06:34:39 UT and (b) beginning at 06:43:46 UT and (c) ending at 07:01:23 UT.

\section{Case 4: Nothern hemisphere on 1 November 1978 (Day 305)}

The next magnetopause crossing examined here occurred on 1 November 1978 (Day 305) between 13:45 and 16:15 UT at 12:00 LT, while ISEE-1 was on an inbound pass. The magnetopause was crossed at around 15:15 UT when the satellite was located at GSE $X=10.9 R_{E}, Y=0.2 R_{E}$, and $Z=0.6 R_{E}$ (Fig. 3). From 13:45 UT until approximately $15: 15 \mathrm{UT}$, the spacecraft was located in the magnetosheath. When the detailed three-dimensional distributions are reconstructed for this period, a number of episodes of northern hemispheric PADs are seen. Components of the magnetic field and the PAD colour-coded index from the P1 channel are displayed in Fig. 10. Figure 11 shows a 15 panel angle/angle plot of the particle flux measured by the P1 channel for the period from 14:48:20 UT to $14: 56: 51 \mathrm{UT}$. Note that while the particle intensity varied during this $9 \mathrm{~min}$ period, the distribution was maintained and controlled by the magnetic field direction. As seen by the colour-coded bar located at the bottom of Fig. 10, the northern hemispheric pitch-angle distribution was observed from 13:52 UT to 15:00 UT. These distributions occurred continuously for periods of time lasting from about 2 to 20 min on three separate occasions during this pass. The lengthy occurrence of this pitch-angle distribution provides strong evidence that a source of energetic particles was located at high-latitudes. The interplanetary conditions measured upstream by the ISEE-3 spacecraft demonstrated an IMF with steady, all positive components similar to that of ISEE-1 shown in Fig. 10 and a solar wind velocity slightly less than $400 \mathrm{~km} / \mathrm{s}$. As the spacecraft approached the magnetosphere, it encountered the current layer associated with the magnetopause. Initial contact with the magnetosphere, starting at 14:34 UT, produced an oscillating and enhanced magnetic field $B_{Z}$ component, as well as an oscillating and variable $B_{Y}$ component. Around 15:03 UT, $B_{Z}$ suddenly increased to about $60 \mathrm{nT}$ above the fluctuating background $B_{Z}$ field strength of about $35 \mathrm{nT}$. During this brief interval, a non-gyrotropic distribution was observed, which is more indicative of a population of particles seen inside the magnetosphere (Williams, 1979). After about $5 \mathrm{~min}$, $B_{Z}$ decreased, suggesting that the spacecraft was once again in the magnetosheath and from about 15:05 UT to 15:16 UT, the spacecraft resided in the magnetosheath. During this period, the pitch-angle distributions observed from the ISEE-1 spacecraft were a mixture of a beaming magnetosheath distribution and background fluxes. After this period passed, the spacecraft again crossed into the magnetosphere (15:16 UT). This is evident both in the magnetic field data, as $B_{Z}$ was much larger than the other components, as well as the ener- 


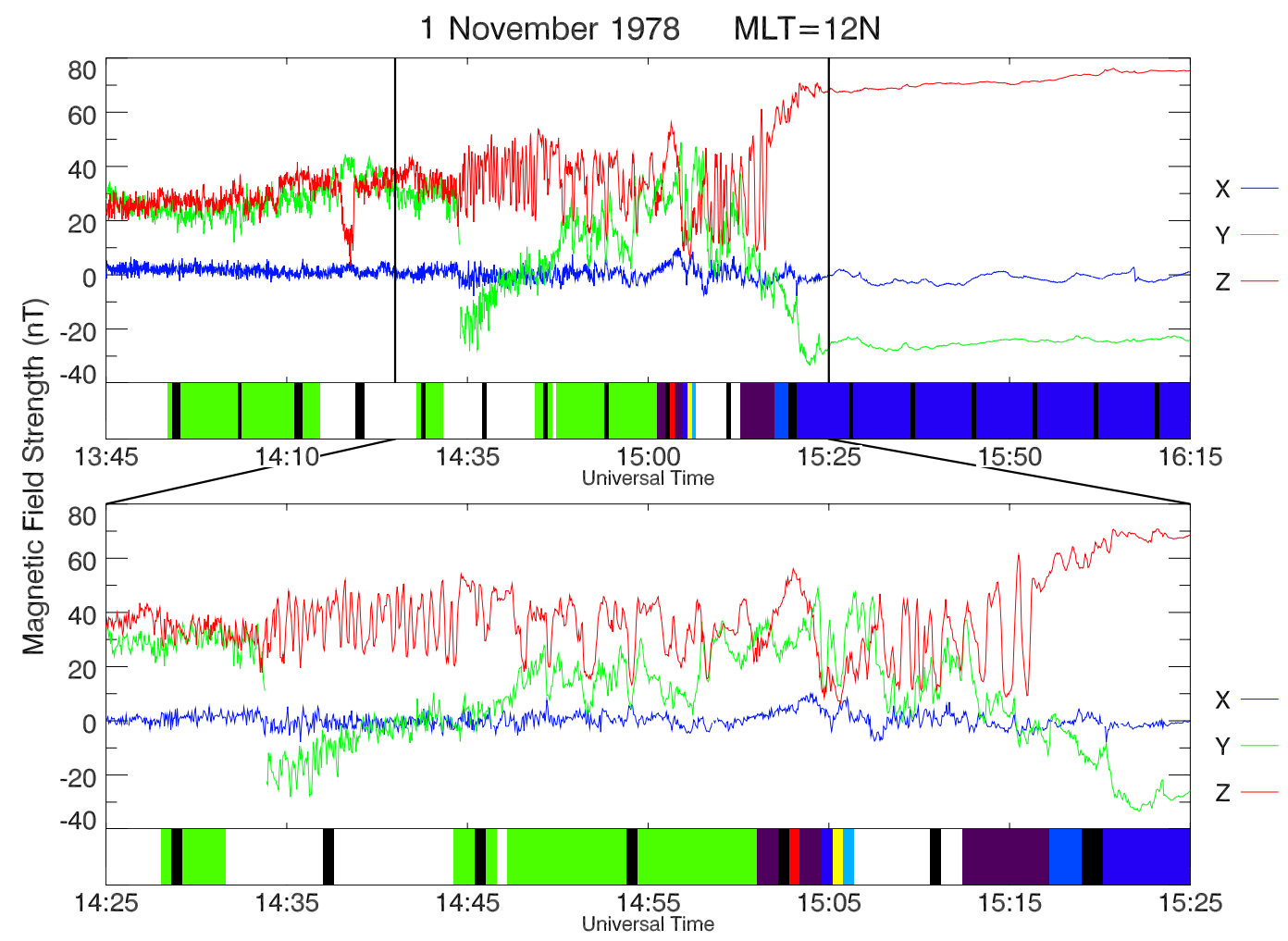

Fig. 10. A plot in the format of Fig. 4 for 1 November 1978 (Day 305) from 13:45 UT to 16:15 UT.

P1 24.0 - $44.5 \mathrm{keV}$ lons 01 November 1978

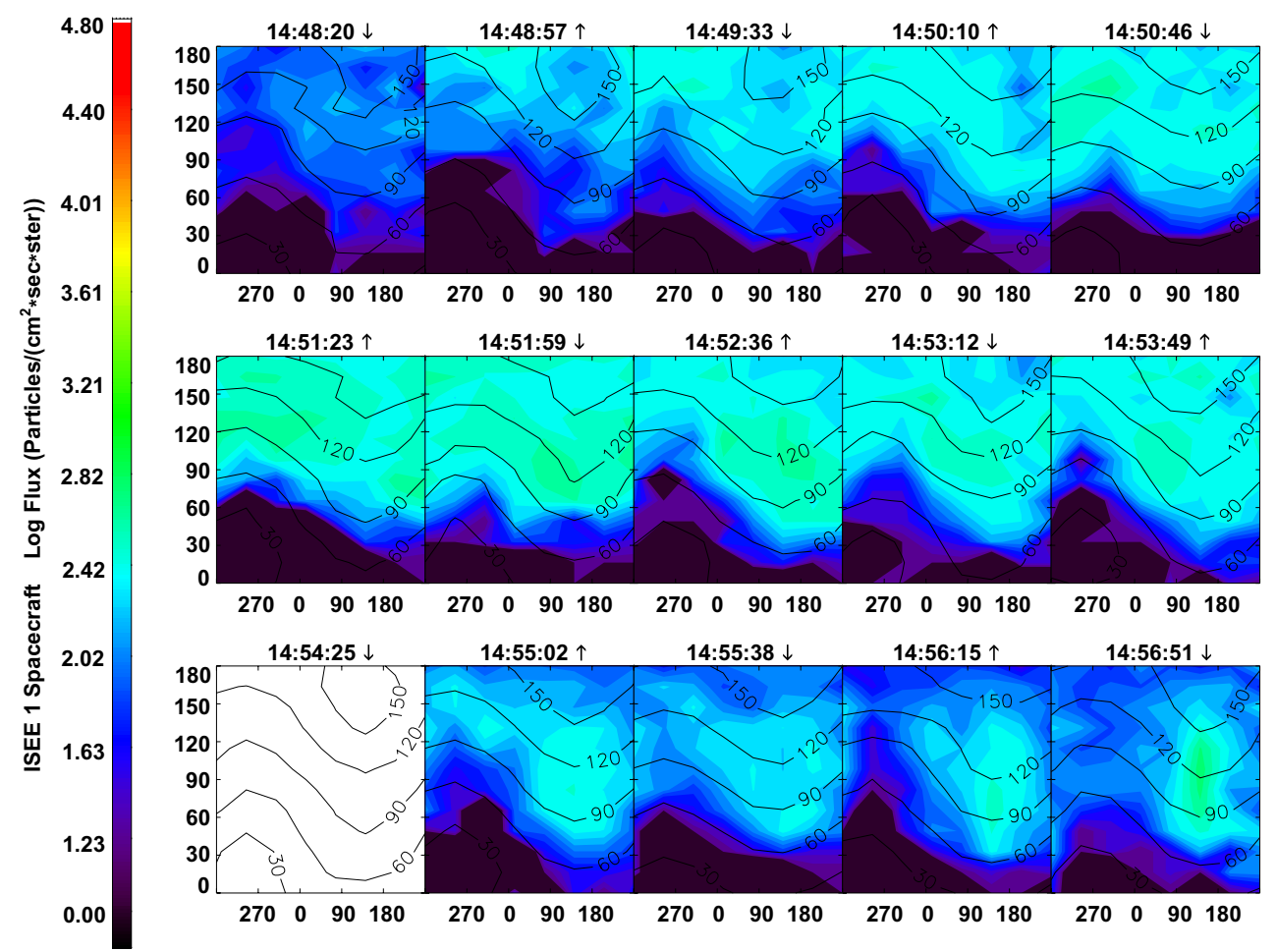

Fig. 11. A time sequence of northern hemispheric PADs for 24 to $44 \mathrm{keV}$ ions on 1 November 1978 (Day 305) from 14:48:20 UT to 14:56:51 UT for the ISEE-1 Medium Energy Particles Experiment P1 channel. 


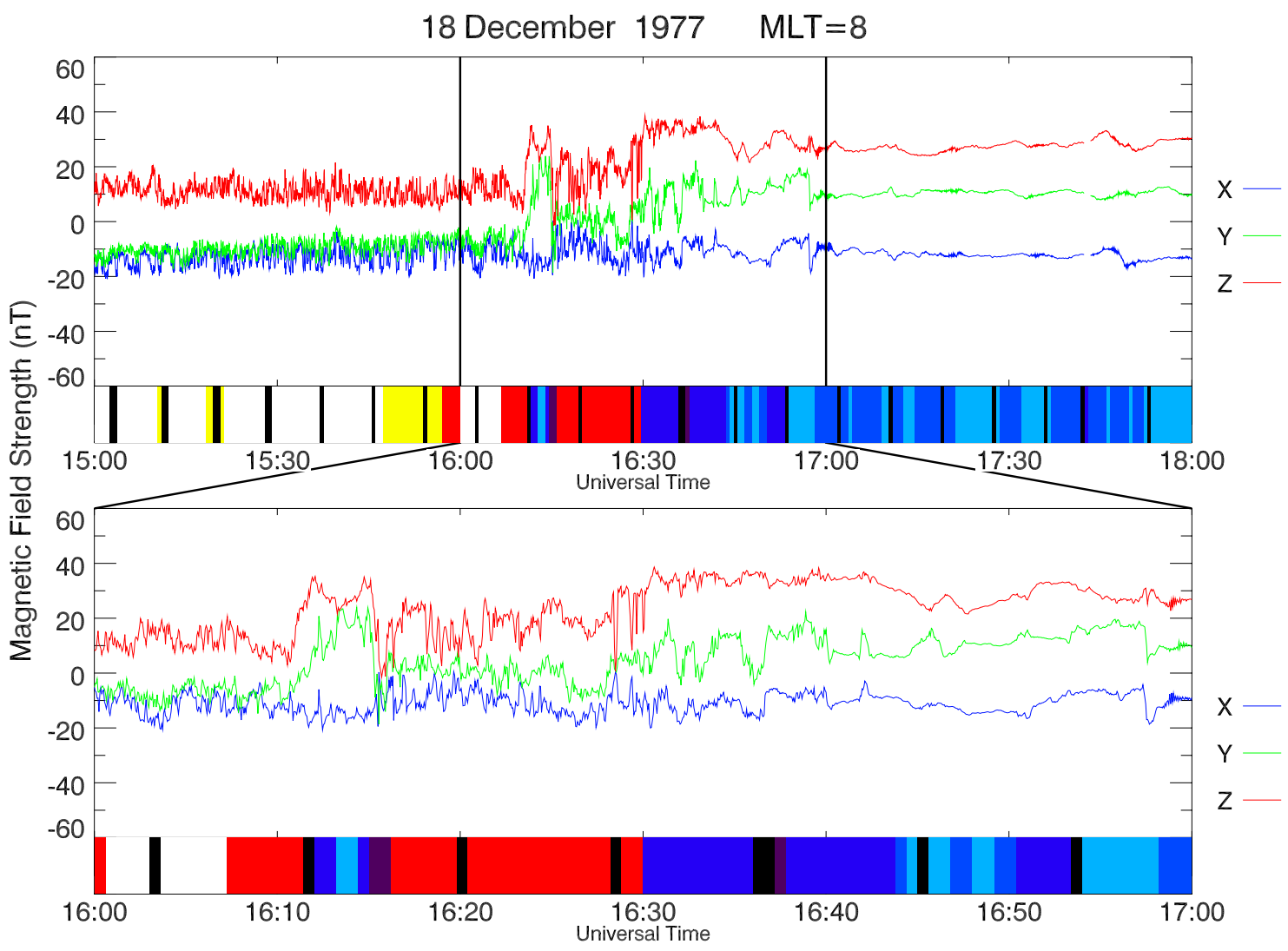

Fig. 12. A plot in the format of Fig. 4 for 18 December 1977 (Day 352) from 15:00 UT to 18:00 UT.

getic particle data, which showed non-gyrotropic, trapped, and isotropic pitch-angle distributions that are commonly associated with the trapping regions of the magnetosphere. Again, the northern hemispheric PADs were observed adjacent to, but sunward of the magnetopause.

\section{Case 5: Southern hemisphere on 18 December 1977 (Day 352)}

ISEE-1 found fluxes of energetic ions coming from the highlatitude southern regions on 18 December 1977 (Day 352) between 15:00 and 18:00 UT, as shown in Fig. 12. ISEE-1 was located at 8:00 LT, four hours away from the subsolar point with GSE coordinates of $X=6.4 R_{E}, Y=-10.5 R_{E}$, and $Z=5.0 R_{E}$ as the satellite crossed through the magnetopause. The magnetosheath field was nominally northward and only the sign of the $B_{Y}$ component changed as the magnetopause was crossed at least three times with the initial crossing at 16:11 UT. Just prior to the magnetopause encounter, the spacecraft remained just outside the magnetosphere and observed large fluxes of energetic ions streaming along field lines from the southern direction. In Fig. 13, pitch-angle distributions are consistently seen, in which the southern hemisphere at the satellite position was completely filled with ions, while the northern hemisphere was empty and these fluxes lasted for an extended period of time. In this case, the southern hemispheric pitch-angle distributions were observed for approximately $5 \mathrm{~min}$ and then for $13 \mathrm{~min}$. During the period between 16:07 UT and 16:30 UT, the ISEE1 satellite crossed the magnetopause at three well-defined times: 16:11 UT, when the spacecraft first encountered the magnetopause (as discussed above) and passed briefly into the magnetosphere; 16:15 UT, as the satellite once again returned to the magnetosheath; and finally at 16:30 UT, when ISEE-1 moved again into the magnetosphere and remained there for the rest of the three hour period. During each of the periods when the spacecraft was located in the magnetosheath, just outside magnetosphere, it observed large fluxes of ions. Figures 13a and 13b show the PAD during the first contact with the magnetosphere and subsequent reentry into the magnetosheath. Since the spacecraft was on an inbound pass, it remained very close, although just outside the magnetosphere for the $15 \mathrm{~min}$ between the second and third magnetopause crossings. As a result, the spacecraft observed continuous fluxes of energetic ions coming only from the southern hemisphere. During the intervals when the spacecraft was inside the magnetosphere, it observed non-gyrotropic pitch-angle distributions near the inner edge of the magnetopause, while trapped and isotropic distributions were seen farther inside. 


\section{P1 24.0 - 44.5 keV lons 18 December 1977}

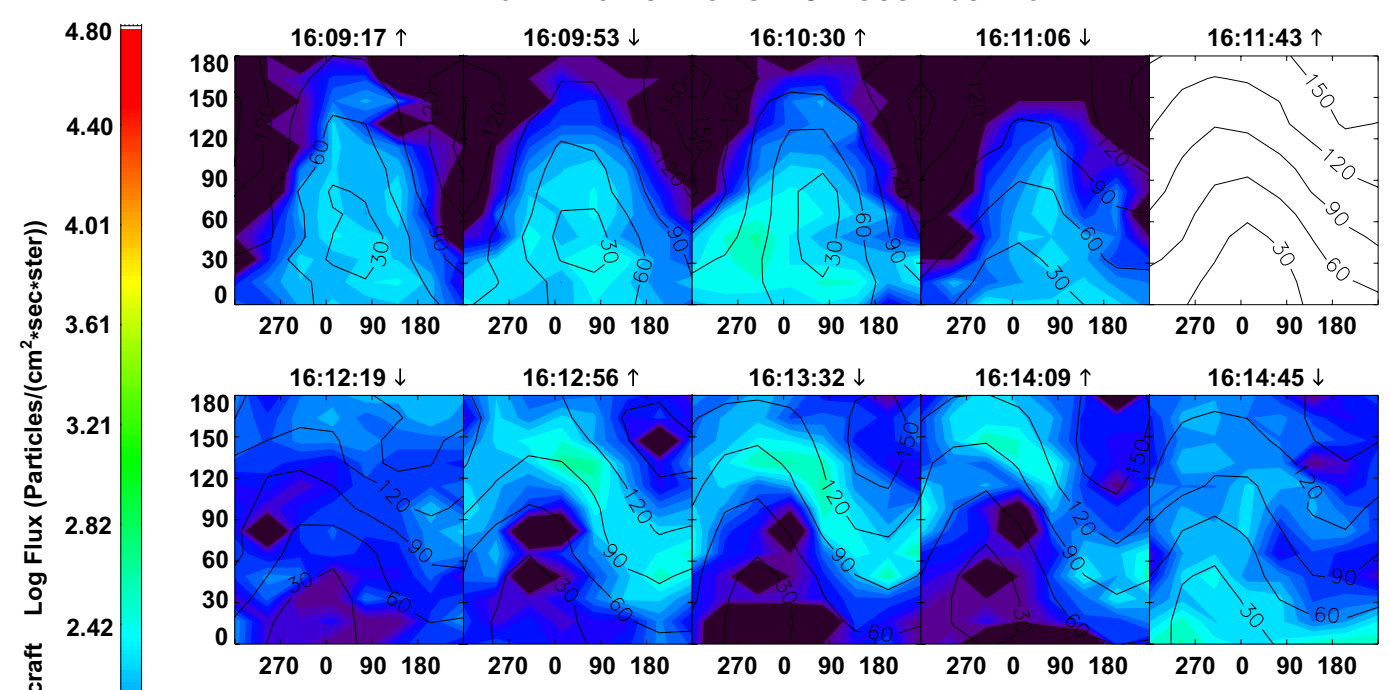

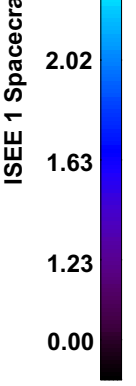

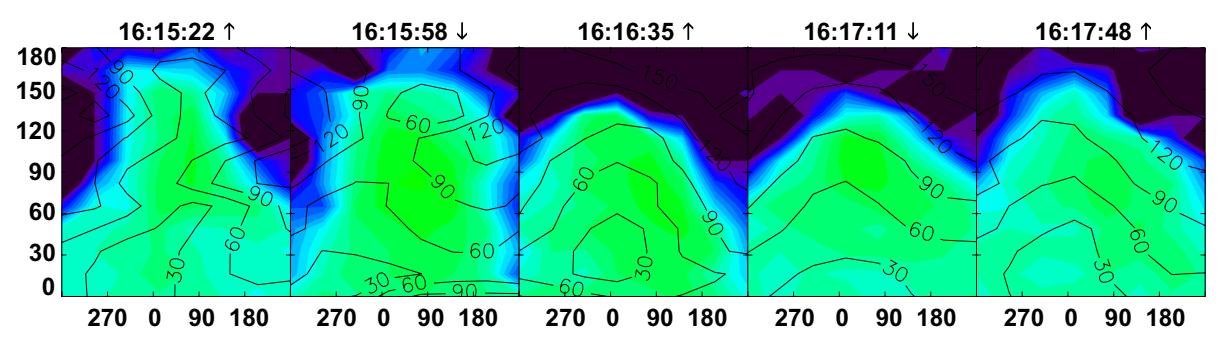

P1 24.0 - $44.5 \mathrm{keV}$ lons 18 December 1977
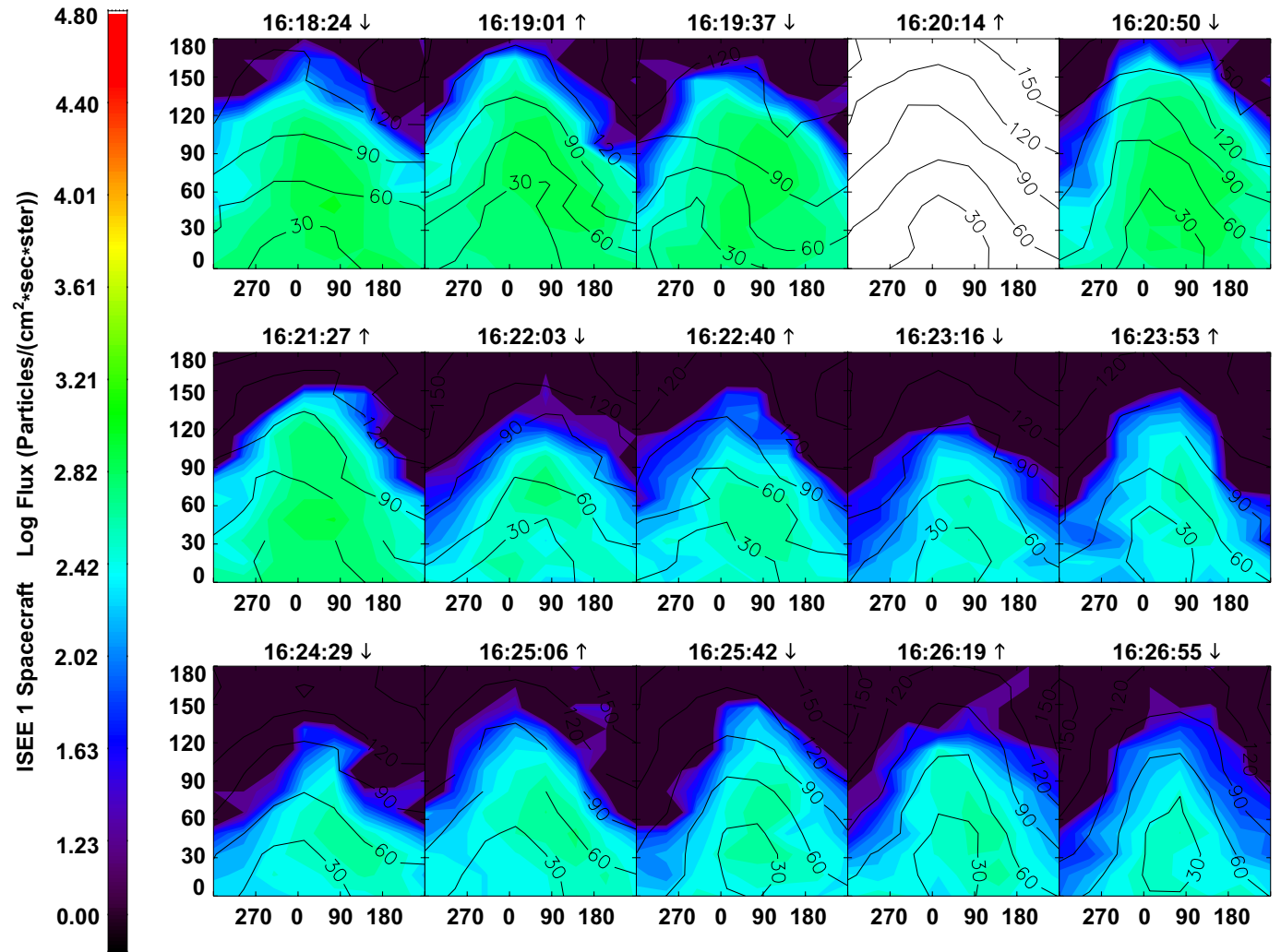

Fig. 13. A time sequence of southern hemispheric PADs for 24 to $44 \mathrm{keV}$ ions on 18 December 1977 (Day 352) from (a) 16:09:17 UT to 16:17:48 UT and (b) 16:18:24 UT to 16:26:55 UT for the ISEE-1 Medium Energy Particles Experiment P1 channel. 


\section{General aspects of these PADs}

For those periods of broad northern hemispheric or southern hemispheric PADs observed just outside the magnetopause, the same type of distribution was observed in energy channels up to at least the P5 energy channel that corresponded to proton energies between $120 \mathrm{keV}$ to $210 \mathrm{keV}$. During these times, the energetic electrons at all energies above $20 \mathrm{keV}$ essentially only appeared once the satellite crossed from the magnetosheath into the magnetosphere.

\section{Conclusion}

In the 5 cases presented here, in a seasonal, chronological order, the ISEE-1 MEPE observed field-aligned fluxes of energetic ions just sunward of the magnetopause near the subsolar point, as the spacecraft transitioned from a region of open field line geometry to a closed, or trapped, geometry. The particle distributions were observed to be present for extended periods on open field lines, ranging from two to tens of minutes, which suggests that they are a common occurrence. Four of the cases used here were from consecutive ISEE-1 orbits through the subsolar magnetopause region, which again further supports their frequent presence. The particles were seen either coming from the northern or southern cusp direction, but were basically never intermixed. The purpose of this study has been accomplished. The type of energetic particle PAD that is predicted by the existance of a possible acceleration mechanism active in the cusp has been observed. There are at least three other possible mechanisms that have been discussed for producing energetic particles upstream of the magnetopause. These are (1) leakage of trapped particles drifting in longitude that impact the magnetopause prior to reaching local noon, (2) acceleration of particles at the bow shock that find their way to the cusp and are reflected back into the magnetosheath, and (3) reconnection by antiparallel magnetic fields at the magnetopause.

For (1), the observation of these broad hemispheric PADs centered along the magnetic field in cases 2 and 5 at local times of 8 and $8.8 \mathrm{~h}, 4 \mathrm{~h}$ away from local noon, argues against leakage of magnetospheric particles from the trapping region of the magnetosphere. Note that these distributions have fluxes with locally mirroring pitch-angles. Equatorially mirroring energetic ions drift westward and follow contours of constant $B$. They will have their closest approach to the magnetopause at the subsolar point, as illustrated in Fig. 14 borrowed from Lyons and Williams (1984). If such particles are present on drift paths that intersect the magnetopause, then they will be preferentially lost to the magnetosheath along the afternoon flank. Any energetic ion that survives its drift through local noon will move further and further away from the magnetopause as it drifts through the dawn quadrant. Both distribution types occurred for extended periods of time just outside of the magnetopause, but the distributions described in cases 2 and 5 above did so even at a local time four hours away from a point where the magnetopause can
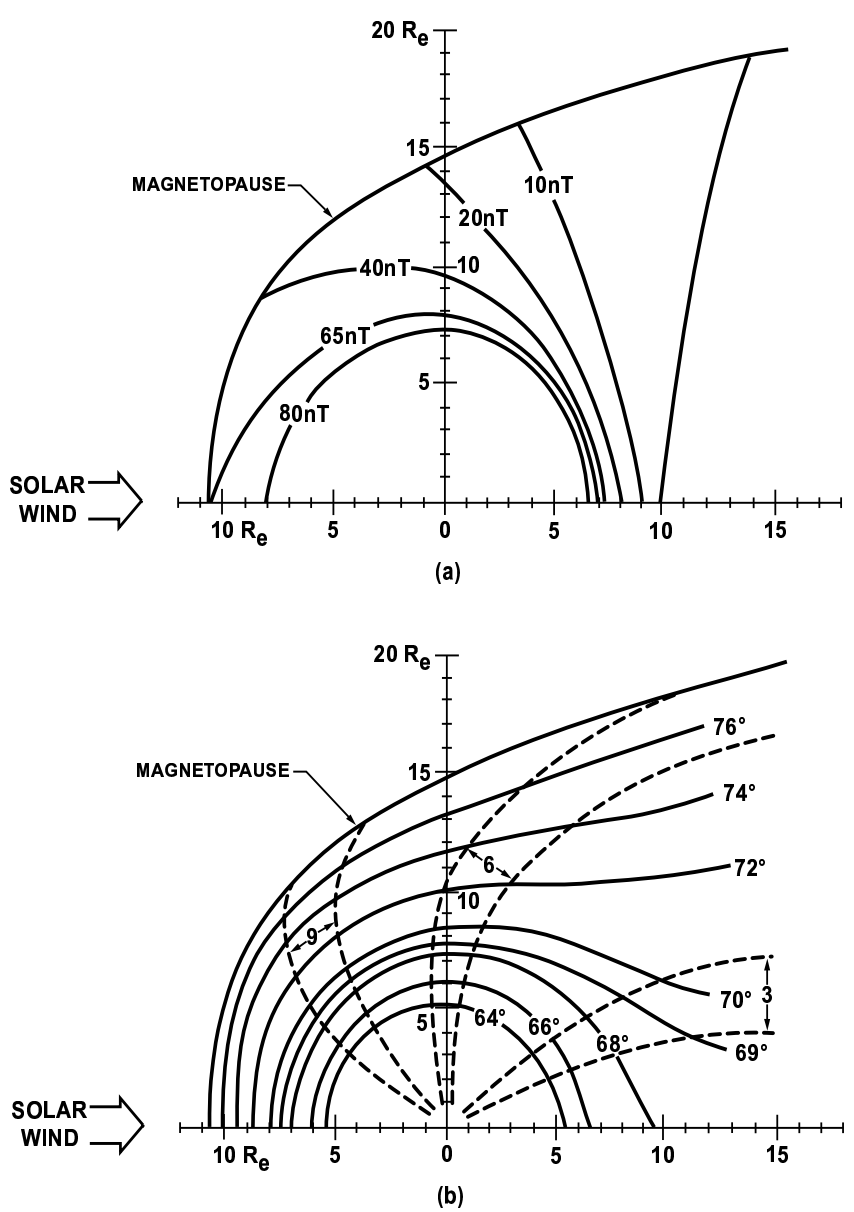

Fig. 14. Average magnetic field characteristics projected onto the geomagnetic equatorial plane: (a) contours of constant magnetic field intensity; (b) contours in the equatorial plane of the latitude and local time of the point of intersection of a field line with the Earth's surface. An energetic particle with an equatorial pitch-angle of $90^{\circ}$ will follow the constant $B$-field contours of (a), whereas a particle with a small equatorial pitch-angle mirroring just above the atmosphere of the Earth will essentially follow the solid contours in (b) as each of these particle types drift in azimuth (from Lyons and Williams, 1984).

impact the gradient drifting locally mirroring ions. The fact that the distributions in cases 2 and 5 had significant fluxes near pitch-angles of $90^{\circ}$ and looked like the distributions of local noon suggests that they are probably not an artifact of leakage of the magnetospheric ring current ions.

For (2), the observation of these broad hemispheric PADs centered on the field line direction being on or just outside of the magnetopause would suggest that they are not produced at the bow shock. A bow shock source would favor their observations at the bow shock or throughout the magnetosheath, rather than just at the magnetopause.

For (3), the subsolar reconnection process is not usually prescribed as a mechanism for producing tens or hundreds of $\mathrm{keV}$ energetic ions. These broad hemispheric PADs centered on a field direction are observed for both northward (cases 4 
and 5) as well as southward (cases 1, 2, and 3) IMF conditions, arguing that reconnection is an unlikely cause of these PADs. Furthermore, if these energetic ions were produced at a subsolar site of reconnection and then streamed away from the $X$-line, they would not preserve their pitch-angle distribution through the field reversal region as these ions do. In fact, they would do just the opposite. Therefore, we can rule out a subsolar reconnection process as the cause for these observed PADs.

Therefore, with these arguments against other possible sources and the direct common observation of the type of distribution predicted by a source located in the high-latitude cusp, it is highly likely that these ions are produced by such a source. Haskell (1969) may have observed highly anisotropic distributions of this type using a geiger tube experiment on Explorer 12. His measurements were interpreted as highly anisotropic $>40 \mathrm{keV}$ electrons just outside the magnetopause, although the sensors were sensitive to very energetic protons above $830 \mathrm{keV}$. We now plan to conduct a more extensive survey of the dayside magnetopause in order to establish in a more quantitative manner the frequency of occurrence of these particular pitch-angle distributions at the magnetopause.

Acknowledgements. The authors have benefited from the efforts of and discussions with the following individuals in the preparation of this manuscript: D. Matthews, M. Alothman, G. Gugliotti, J. Bhattacharjya, E. Beiser, J. Sanborn, all at Boston University. The authors thank Dr. J. A. Van Allen for pointing out the prior work by G. Haskell.

Topical Editor G. Chanteur thanks T. Eastman and another referee for their help in evaluating this paper.

\section{References}

Alothman, M.: Observation and Modeling of the Trapping Boundary of the Radiation Belts, Ph. D. Disertation, Boston University, 2000.

Baker, D. N. and Stone, E. C.: The Magnetopause Energetic Electron Layer 1. Observations Along the Distant Magnetotail, J. Geophys Res., 83, No. A9, 4327, 1978.
Chen, J. and Fritz, T. A.: Correlation of cusp MeV helium with turbulent ULF power spectra and its implications, Geophys. Res. Lett., 25, 4113-4116, 1998.

Chen, J., Fritz, T. A., Sheldon, R. B., Spence, H. E., Spjeldvik, W. N., Fennell, J. F., and Livi, S.: A new temporarily confined population in the polar cap during the August 27, 1996 geomagnetic field distortion period, Geophys. Res. Lett., 24, 1446, 1997.

Chen, J., Fritz, T. A., Sheldon, R. B., Spence, H. E., Spjeldvik, W. N., Fennell, J. F., Livi, S., Russell, C. T., Pickett, J. S., and Gurnett, D. A.: Cusp energetic particle events: Implication for a major acceleration region of the magnetosphere,J. Geophys. Res., 103, A1, 69, 1998.

Fritz, T. A. and Chen, J.: The cusp as a source of magnetospheric particles, Radiation Measurements, 30, 5, 599-608, 1999.

Haskell, G.: Anisotropic fluxes of energetic particles in the outer magnetosphere, J. Geophys. Res, 74, 7, 1740, 1969.

Lyons, L. R. and Williams, D. J.: Quantative Aspect of the Magnetospheric Physics, D. Reidel Pub. Co, Hingham, MA, 231, 1984.

McIlwain, C. E.: Coordinates for Mapping the Distribution of Magnetically Trapped Particles, J. Geophys. Res., 66, 11, 3681-3691, 1961.

Meng, C. I. and Anderson, K. A.: A Layer of Energetic Electrons (> $40 \mathrm{keV}$ ) Near the Magnetopause, J. Geophys. Res., 75, 1827, 1970.

Mitchell. D. G., Kutchko, F., Williams, D. J., Eastman, T. E., Frank, L. A., and Russell, C. T.: An extended study of the lowlatitude boundary layer on the dawn and dusk flanks of the magnetosphere, J. Geophys. Res., 92, A7, 7394, 1987.

Russell, C. T.: The ISEE 1 and 2 fluxgate magnetometers, IEEE Trans. Geosci. Electr., GE-16, 239-242, 1978.

Sarris, E. T., Krimigis, S. M., and Armstrong, T. P.: Observation of Magnetospheric Bursts of High-energy Protons and Electrons at 〜 35 R with IMP 7, J. Geophys Res., 81, A13, 2341, 1976.

Williams, D. J.: Magnetopause Characteristics Inferred from ThreeDimensional Energetic Particle Distributions, J. Geophys. Res., 84, A1, 101-104, 1979.

Williams, D. J., Keppler, E., Fritz, T. A., Wilken, B., and Wibberenz, G. W.: The ISEE-1 and 2 medium energy particles experiment, IEEE Trans. Geosci. Electr., GE-16, 270-278, 1978.

Williams, D. J., Mitchell, D. G., Eastman, T. E., and Frank, L. A.: Energetic particle observation in the low-latitude boundary layer, J. Geophys. Res., 90, 5097, 1985. 